\title{
Reuna
}

\section{SELF-CHECKOUT NO VAREJO: IMPLICAÇÕES NA SATISFAÇÃO DOS CONSUMIDORES}

\section{SELF-CHECKOUT IN RETAIL: IMPLICATIONS IN CONSUMER SATISFACTION}

\author{
http://dx.doi.org/10.21714/2179-8834/2019v24n1p41-57
}

\author{
Gustavo Nunes Maciel \\ Universidade Federal de Lavras (UFLA), Brasil. \\ E-mail: gustavonunesmaciel@yahoo.com.br \\ José Augusto Oliveira \\ Universidade Federal de Lavras (UFLA), Brasil. \\ E-mail: jaugusto.oliveira@outlook.com
}

\begin{abstract}
Gustavo Clemente Valadares
Instituto Federal do Sul de Minas Gerais (IFSULDEMINAS) Campus Passos e Universidade Federal de Lavras (UFLA), Brasil.

E-mail: gustavo.gvmkt@gmail.com
\end{abstract}

Paulo Henrique Montagnana Vicente Leme

Universidade Federal de Lavras (UFLA), Brasil.

E-mail: lemeph@gmail.com

\author{
Daniel Carvalho Rezende \\ Universidade Federal de Lavras (UFLA), Brasil. \\ E-mail: rezendedc@gmail.com
}

Submissão: 21 Dez. 2018 Publicação: 25 Fev. 2019. Sistema de avaliação: Double blind review.
Centro Universitário UNA, Belo Horizonte - MG, Brasil. Editor geral: Prof. Dr. Gustavo Quiroga Souki

Este artigo encontra-se disponível nos seguintes endereços eletrônicos:

http://revistas.una.br/index.php/reuna/article/view/1059

http://dx.doi.org/10.21714/2179-8834/2019v24n1p41-57

\section{Resumo}

A dinâmica da concorrência nos mercados contemporâneos aliada ao surgimento de inovações tecnológicas traz às organizações um desafio constante de sobrevivência frente à intensa competitividade empresarial. Assim, buscando a diferenciação e a permanência nos mercados, percebe-se que alguns empresários apostam em recursos tecnológicos que vêm transformando a interação entre os consumidores e os varejistas. Neste contexto, o serviço de autoatendimento surge no setor supermercadista para reduzir os custos e otimizar o tempo de filas. Por consequência, gestores têm percebido em suas rotinas resultados positivos quanto à experiência e satisfação dos clientes na utilização dos terminais de autoatendimento. O presente trabalho tem como objetivo investigar a relação do consumidor com o serviço de autoatendimento empregado por uma rede supermercadista da cidade de Lavras, no sul de Minas Gerais. Entre os resultados encontrados, fatores como maior comodidade, maior rapidez e a fila percebida nos caixas tradicionais, assumiram a posição de maior relevância para os entrevistados. Esta pesquisa traz contribuições em âmbito acadêmico, ao discutir as implicações do serviço de autoatendimento na satisfação dos consumidores; e em âmbito gerencial, 
contribuindo com os gestores no processo de implantação dos terminais de autoatendimento ao apresentar os fatores que motivam a decisão dos clientes em utilizar o serviço.

Palavras-chave: Autoatendimento; Self-checkout; Satisfação do consumidor.

\section{Abstract}

The dynamics of competition in contemporary markets coupled with the emergence of technological innovations bring organizations a constant challenge of survival in the face of intense business competitiveness. Thus, seeking differentiation and permanence in the markets, it is noticed that some entrepreneurs bet on technological resources that are transforming the interaction between consumers and retailers. In this context, the self-service service appears in the supermarket sector to reduce costs and optimize queuing time. Consequently, managers have perceived in their routines positive results regarding the experience and satisfaction of customers in the use of self-service terminals. The present work aims to investigate the relationship of the consumer with the self-service service employed by a supermarket chain in the city of Lavras, in the south of Minas Gerais. Among the results found, factors such as greater convenience, greater speed and the queue perceived in the traditional boxes, assumed the position of greater relevance for the interviewees. This research brings contributions in the academic scope when discussing the implications of the service of self-service in the satisfaction of the consumers; and in the managerial context, contributing with the managers in the process of implementation of the self-service terminals, presenting the factors that motivate the customers' decision to use the service.

Keywords: Self-Service; Self-checkout; Consumer satisfaction.

\section{Introdução}

O movimento dinâmico vivenciado no mercado, gerado pelos processos de globalização aliados ao avanço intermitente da tecnologia, traz às organizações contemporâneas um cenário de instabilidade onde a busca por alternativas para se manterem competitivas se torna fundamental (BALBIM JUNIOR; BORNIA, 2011; BARRETO FERNANDES; ORTUÑO, 2017; DEMIRCI OREL; KARA, 2014; OLIVEIRA et al., 2017; TRIERWEILLER et al., 2017). Neste desafio, as organizações necessitam romper paradigmas até então estabelecidos com a antecipação de recursos tecnológicos que possibilitem agregar valor aos produtos e serviços e, portanto, se tornar diferenciada no segmento (TRIERWEILLER et al., 2017). Complementarmente, Johnson et al. (2019) enfatiza que para a sobrevivência em um contexto tão competitivo é necessário que as organizações compreendam melhor o valor que seu negócio traz para o consumidor.

Todavia, a evolução tecnológica vem transformando energicamente a interação entre os consumidores e os varejistas (BARRETO FERNANDES; ORTUÑ̃, 2017; DEMIRCI OREL; KARA, 2014). De acordo com Inman e Nikolova (2017), a capacidade tecnológica que o varejo dispõe atualmente nunca foi tão 
grande, tornando suas atividades cada vez mais complexas e passíveis de lucratividade.

Como um dos fatores mais preocupantes em lojas físicas está a qualidade de conforto do ambiente, ou seja, locais como supermercados e lojas de departamentos, que possuem altos fluxos de movimento durante todo o dia, ou em determinados horários, sofrem com este preocupante problema (OLIVEIRA et al., 2017). No entanto, para reverter essa e outras situações que afetam negativamente a experiência dos consumidores, a busca por vantagens competitivas levaram os varejistas a utilizar novos recursos tecnológicos, a partir de meios de telecomunicações e tecnologias de informação, a fim de fornecer produtos e serviços aos consumidores e melhorar a interação entre o varejo e seus consumidores (LENG; WEE, 2017).

Desde os anos 90, serviços de autoatendimento são oferecidos em diversos tipos de negócio como quiosques de atendimento, lanchonetes, postos de gasolina, entre outros (DEMIRCI OREL; KARA, 2014, JOHNSON et al., 2019; LENG; WEE, 2017). Vale destacar que o mercado global de tecnologia de recursos de autoatendimento deverá acumular o valor de US\$31,75 bilhões até o ano de 2020 (ALLIED MARKET RESEARCH, 2015).

Entre os variados tipos de estabelecimentos que utilizam este recurso tecnológico, algumas redes de supermercado têm chamado a atenção pelo emprego de uma tecnologia de auto atendimento, termo traduzido de Self-Service Tecnology (SST) (DEMIRCI OREL; KARA, 2014). Com este aparato, o consumidor torna-se independente para registrar seu produto, ensacá-lo e, posteriormente, realizar o pagamento sem a interação com qualquer funcionário.

Um dos principais motivos das redes supermercadistas oferecerem o serviço de autoatendimento é para reduzir os custos e otimizar o tempo de filas (KOKKINOU; CRANAGE, 2013), porém, alguns gestores têm auferido em suas vivências resultados positivos quanto à experiência do cliente e sua satisfação em utilizar os terminais (DEMIRCI OREL; KARA, 2014). Neste sentido, a satisfação está atrelada a fatores que excedem as competências técnicas do produto, como na qualidade dos serviços prestados (TRIERWEILLER et al., 2017).

A satisfação dos consumidores é considerada fator determinante para a empresa se firmar no mercado e sobreviver aos percalços nele encontrados (BALBIM JUNIOR; BORNIA, 2011). Complementarmente, ainda segundo os autores, os clientes satisfeitos tendem a retribuir a lealdade, realizar novas compras e repercutir a imagem do estabelecimento de forma positiva. De acordo com Grewal e Levy (2007), independentemente de um cliente optar por um varejista específico torna-se possível atraí-lo por diversos fatores como uma maior gama de produtos, programas de fidelidade e por serviços inovadores.

Neste contexto, as investigações acerca da satisfação dos clientes consistem num recurso oportuno para a manutenção da relação entre o varejo e o cliente (TRIERWEILLER et al., 2017). Segundo Leng e Wee, investigações anteriores apontaram que as variáveis demográficas e psicográficas juntamente com a avaliação da tecnologia pelos consumidores podem ser fatores determinantes para os consumidores optarem pelo self-checkout. 
As pesquisas que envolvem as tecnologias de autoatendimento ganharam abrangência no cenário mercadológico e acadêmico, especialmente a adesão deste recurso tecnológico no ambiente do varejo (FERNANDES; PEDROSO, 2017; LEE; LYU, 2016). Especificamente no Brasil, a adoção de serviços de autoatendimento em supermercados ainda é muito recente e carece de uma maior observação. Além disso, novas investigações sobre a relação entre os serviços de autoatendimento e os consumidores são necessárias e rogadas por outros pesquisadores da temática (COLLIER; BREAZEALE; WHITE, 2017; LEE; LYU, 2019).

Por isso, o presente artigo tem como objetivo geral investigar o perfil e os fatores relacionados à satisfação dos consumidores que optam por utilizar o serviço de autoatendimento empregado por uma rede supermercadista da cidade de Lavras, no sul de Minas Gerais. Como objetivos específicos espera-se analisar a satisfação do consumidor quanto à experiência em utilizar o novo equipamento; verificar os fatores que mais influenciam na sua escolha; e, finalmente, confrontar com a literatura acerca do tema, apresentando os pontos divergentes e em comum.

A estrutura do presente trabalho é composta por seis capítulos. Após a introdução, no segundo capítulo será exposta a fundamentação teórica, o terceiro abordará os aspectos metodológicos, o quarto apresentará a análise dos dados e discussão, o quinto as considerações finais e, por fim, no sexto capítulo serão elencadas as referências bibliográficas.

\section{Fundamentação teórica}

\subsection{Self-checkout}

Os recursos tecnológicos de autoatendimento, traduzidos da expressão SelfService Tecnology (SST), modificaram as relações cotidianas do consumidor nos mais diversos setores do mercado e tornaram-se onipresentes no cotidiano contemporâneo (CONSIDINE; CORMICAN, 2016; DEMIRCI OREL; KARA, 2014). De acordo com Mukerjee, Deshmukh e Prasad (2019), a SST refere-se às atividades e/ou os benefícios adquiridos a partir e em torno da tecnologia.

Entretanto, a incorporação deste tipo de recurso nos ambientes comerciais deve acompanhar a evolução da qualidade do serviço prestado pela organização e os cuidados necessários de implementação, promovendo o incremento da prestação ao cliente e, por sua vez, garantindo a satisfação e a possível intenção de retorno ( COLLIER; BREAZEALE; WHITE, 2017; CONSIDINE; CORMICAN, 2016; FERNANDES; PEDROSO, 2017).

O sistema self-checkout consiste em um tipo específico de SST e possui o potencial de otimizar a produtividade e eficiência a partir de um processo automatizado (KAZANCOGLU; KURSUNLUOGLU YARIMOGLU, 2018). Ainda segundo os autores, tal aparato tecnológico é uma forma inovadora de SSTs e possibilita que os varejistas diferenciem seus serviços aos concorrentes e a percepção de seus clientes.

Seguindo a tendência, algumas redes de supermercado estão aderindo sistemas de autoatendimento que possibilitam ao consumidor realizar as operações de registro do produto, ensacamento e pagamento sem a interação com um atendente humano (DEMIRCI OREL; KARA, 2014). O dispositivo chamado self- 
checkout é considerado uma das versões mais disseminadas dentro da tecnologia de autoatendimento e a sua implementação fornece ao consumidor uma alternativa aos caixas tradicionais com operadores (BARRETO FERNANDES; ORTUÑO, 2017).

De acordo com Meuter et al. (2000), os serviços de autoatendimento podem ser definidos como interfaces tecnológicas que possibilitam ao consumidor produzir um serviço independente da interação direta com funcionários de um estabelecimento. Estes equipamentos dão ao comerciante a possibilidade de estabelecer diferentes combinações a partir de variados módulos, favorecendo ao alinhamento do recurso às necessidades dos consumidores que o utilizará (BARRETO FERNANDES; ORTUÑO, 2017). Ressalta-se que o sistema deve ser de fácil operação e possuir natureza intuitiva, permitindo que o usuário aprenda de forma clara e recorde facilmente do processo de utilização nas próximas compras.

Devido a sua natureza, as tecnologias de autoatendimento demandam participação ativa do cliente para que o serviço seja realizado (DEMIRCI OREL; KARA, 2014). Por isso, torna-se necessário que o consumidor tenha motivação em realizar esta ação, a partir do reconhecimento de um benefício ao optar pelo recurso (FERNANDES; PEDROSO, 2017). Entre as maiores necessidades vivenciadas pelos consumidores atuais está a escassez de tempo, proveniente da alta carga de seus afazeres, como estudar, trabalhar, cuidar da família, etc.; neste sentido, os equipamentos são direcionados para satisfazerem esta carência latente, de uma população que vai às compras frequentemente e, em muitos casos, compram poucas unidades de cada vez (BARRETO FERNANDES; ORTUÑO, 2017).

Com relação à espera em filas, percebe-se que quanto mais tempo os consumidores aguardam por um serviço, menor o nível de satisfação percebido (FERNANDES; PEDROSO, 2017). Assim, a avaliação da experiência de um sistema de autoatendimento poderá ser favorável ou não, dependendo da maneira como a experiência será percebida pelo cliente (DJELASSI; DIALLO; ZIELKE, 2018). Contudo, nota-se que a adoção do sistema tecnológico no varejo pode trazer valiosos resultados como vantagem competitiva e a expansão da base de clientes (FERNANDES; PEDROSO, 2017).

\subsection{Satisfação do consumidor}

A satisfação do consumidor é um tema global bastante relevante na área de marketing; em nível nacional, esta constatação se mantém e apresenta contínua evolução tanto no escopo teórico quanto no escopo gerencial (TRIERWEILLER et al., 2017). De forma complementar, percebe-se que a investigação acerca do tema é fundamental para a melhoria dos serviços ofertados pelas empresas, contribuindo diretamente para permanência destas no mercado.

Portanto, a satisfação do consumidor requer atenção no contexto mercadológico, por impactar diretamente na imagem do negócio, especialmente para gestores de mercados varejistas, devido ao resultado percebido do cliente satisfeito em retornar e promover à loja (ANDERSON; FORNELL; LEHMANN, 1994; FORNELL et al., 1996; (OLIVEIRA et al., 2017).

De acordo com Balbim, Júnior e Bornia (2011), a satisfação é resultante da percepção e tradução das necessidades do consumidor em relação aos atributos do 
produto ou serviço. Além disso, ela é considerada como base para a compreensão dos comportamentos posteriores do indivíduo como a lealdade, a recompra e a propaganda (TRIERWEILLER et al., 2017). Entretanto, conforme os autores, por se tratar de um constructo subjetivo, a satisfação é observada como um desafio para os gestores organizacionais, devido suas características dinâmica e imensurável.

A satisfação do consumidor é vista como feedback do contentamento percebido pelo consumidor a partir do consumo de um bem ou serviço, podendo ter nível alto ou baixo (OLIVER, 1997). Vale acrescentar que o referido grau é resultado da comparação do consumidor acerca do desempenho que a experiência ofereceu (TRIERWEILLER et al., 2017); e o cliente considerado satisfeito remete-se aquele que depreende o atendimento de suas necessidades, de forma igual ou além do que se desejava.

A partir da compreensão do grau de satisfação do consumidor, é possível reconhecer a potencialidade de fidelização; isto porque clientes leais promovem, confiam e indicam novos fregueses para o negócio (BALBIM JUNIOR; BORNIA, 2011). No contexto supermercadista, Prado e Machetti (1997) conferem que a verificação da satisfação dos consumidores é encarada como fator de êxito, devido à possibilidade de readequação das ações de marketing mediante a análise da satisfação.

É importante destacar que a lealdade possui como antecedente no comportamento do consumidor a satisfação; neste sentido, ela exerce influência positiva no processo de recompra quanto na promoção da marca ou do estabelecimento (GRONROOS, 1993).

\section{Metodologia de pesquisa}

Esta pesquisa se caracteriza como quantitativa descritiva, uma vez que teve como objetivo estudar um grupo em específico, com base em técnicas padronizadas para a coleta de dados, além do emprego de análise estatística (GIL, 2008).

Optou-se pelo levantamento de campo como método de pesquisa, técnica esta utilizada para a coleta de dados, a partir de indivíduos, com vasta utilização em situações que requerem uma amostragem de dados (HAIR et al., 2009). Como instrumento para a coleta de dados da pesquisa foi utilizado um questionário auto administrado, estruturado com questões de múltipla escolha, sendo onze delas com base na escala de concordância de cinco pontos (HAIR et al., 2009).

O questionário aplicado foi desenvolvido a partir dos trabalhos de Barreto Fernandes e Ortuño (2017) e Fernandes e Pedroso (2017). Desta maneira, foram utilizados os questionamentos mais relevantes destas pesquisas, acrescido de questionamentos oriundos do contexto ao qual esta pesquisa foi idealizada.

A população do estudo foi composta por clientes de uma rede de supermercados na cidade de Lavras-MG, que utilizaram o sistema de autoatendimento (self-checkout) do estabelecimento ao menos uma vez. A seleção dos respondentes foi realizada utilizando a técnica de amostragem por conveniência. Esta escolha se deu por sua condição dinâmica e por representar baixo custo de aplicação. (HAIR et al., 2009). Cabe ressaltar que a escolha pela amostragem não probabilística por conveniência se deu por ser impossível compreender a população 
total do estudo (MALHOTRA, 2012). A amostra final foi de 200 respondentes válidos. Em relação ao tamanho da amostra, foram seguidas as recomendações de (HAIR et al., 2009) de que, para se trabalhar com técnicas de estatística multivariada, deve-se contar com um número de unidades amostrais equivalente a, pelo menos, dez vezes o número de variáveis de pesquisa.

Os questionários foram aplicados de maneira presencial com os clientes após a utilização do terminal de autoatendimento em um supermercado localizado na cidade de Lavras-MG.

A análise dos dados foi realizada com a utilização do software SPSS. Os dados coletados foram tabulados em uma planilha eletrônica e posteriormente realizadas análises descritivas bem como utilizadas algumas técnicas de análise multivariada como análise fatorial, análise de cluster e análise discriminante, respectivamente. A análise descritiva envolve a manipulação dos dados para então sintetizá-los e descrevê-los, trazendo apenas informações que estejam nos limites reais dos dados (FREUND, SIMON, 2000), enquanto a análise multivariada analisa a dependência ou a interdependência entre as variáveis ou conjunto das variáveis observadas (HAIR et al., 2009).

A análise fatorial foi realizada por meio da técnica de análise dos componentes principais. Esta é uma técnica de redução de dados cujo objetivo principal é a construção de uma combinação linear das variáveis principais que representam a totalidade e contribui para a interpretação das relações existentes entre as variáveis. (HAIR et al., 2009). A confiabilidade dos fatores foi realizada por meio do Alfa de Cronbach.

No intuito de se compreender as similaridades e discrepâncias entre o comportamento dos respondentes foram realizadas as análises de cluster, discriminante e ANOVA. A análise de cluster é realizada para identificar um número pequeno de agrupamentos para um todo, que tem aspectos semelhantes. Em geral o subgrupo homogêneo é baseado nas similaridades dos perfis dos respondentes. Esta análise pode ainda ser utilizada para gerar classes agrupando objetos com base no princípio de maximizar a similaridades intra-classes e maximizar similaridade inter-classes (HAIR et al., 2009). A análise discriminante por sua vez, é indicada quando se tem uma variável (ou mais) que está relacionada com alguma característica da amostra, no intuito de se identificar, dentro do grupo da variável, qual é a tendência de expressar alguma discriminação ou não (MALHOTRA, 2012). Por fim, a utilização da técnica ANOVA permite a comparação de médias entre duas ou mais variáveis independentes (HAIR et al., 2009), favorecendo assim, o processo de caracterização dos clusters observados.

\section{Análise e discussão dos resultados 4.1 Análise Descritiva}

A pesquisa alcançou um total de 200 respondentes. Destes, 101 (50,5\%) são do gênero masculino e $99(49,5 \%)$ do gênero feminino, demonstrando o equilíbrio entre a quantidade de homens e mulheres participantes. Com relação a faixa etária, $62,5 \%$ dos respondentes tem entre 18 e 30 anos, 75,0\% não possuem filhos, 63,0\% são solteiros, 69,0\% não exercem atividade remunerada e 85,4\% cursam ou já concluíram o ensino superior. A figura 01 apresenta a distribuição de frequência das variáveis demográficas da amostra. 


\section{Figura 01: Características demográficas da amostra}

Gênero

Masculino
Feminino

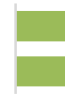

Faixa etária
Exerce atividade remunerada

$\mathbf{5 0 , 5 \%}$

$$
\begin{aligned}
& \text { Sim } \\
& \text { Não }
\end{aligned}
$$

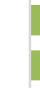
$31,0 \%$

$69,0 \%$

Estado Civil

Entre 18 e 30 anos Entre 31 e 45 anos Entre 46 e 60 anos Acima de 60 anos

\begin{tabular}{|l|l|}
\hline & $62,5 \%$ \\
\hline $24,0 \%$ \\
\hline $11,5 \%$ \\
\hline $2,0 \%$ \\
\hline
\end{tabular}
Solteiro Separado Outro

$63,0 \%$ $29,5 \%$ ,

Número de filhos

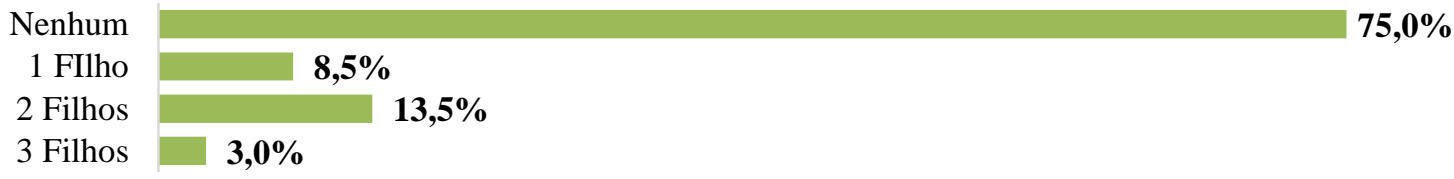

Fonte: Autores (2018)

Segundo os dados da pesquisa, 196 respondentes (98,0\%) acessam a internet diariamente, $2(1,0 \%)$ de uma a cinco vezes por semana e outros $2(1,0 \%)$ não acessam a rede. Quanto a utilização de computadores, $143(71,5 \%)$ fazem uso diário, $26(13,0 \%)$ utilizam o equipamento durante cinco dias da semana, $16(8,0 \%)$ fazem uso em três dias, $10(5,0 \%)$ em uma vez por semana e $2(1 \%)$ não utilizam o equipamento. Grande parte da amostra utiliza frequentemente equipamentos celulares, $194(97,0 \%)$ respondentes utilizam esse tipo de equipamento todos os dias, $5(2,5 \%)$ fazem uso de celulares em cinco dias da semana e $1(0,5 \%)$ respondente utiliza o dispositivo celular apenas uma vez por semana.

Quanto a utilização do self-checkout, $114(57,0 \%)$ dos respondentes não possuíam experiencia anterior na operação do equipamento, enquanto $86(43,0 \%)$ haviam utilizado o dispositivo em, no mínimo, uma ocasião.

Portanto, pode-se concluir que a amostra é composta predominantemente por homens e mulheres jovens, sem filhos, solteiros, que não exercem atividade remunerada, que cursam ou já concluíram o ensino superior, que acessam a internet e utilizam equipamentos eletrônicos diariamente e que não possuíam experiência prévia quanto a utilização de terminais de self-checkout. Tais resultados podem ser compreendidos por pelo menos duas perspectivas: (i) Lavras é reconhecida como uma importante cidade universitária no estado de Minas Gerais, que oferta diferentes cursos técnicos, de graduação e pós-graduação, neste sentido, a cidade concentra um grande número de estudantes; (ii) o fato do equipamento de Self-Checkout ser informatizado, pode atrair o público mais jovem ao passo que pode inibir o público mais velho, que não apresenta tanta disposição em utilizar recursos tecnológicos. 


\subsection{Análise estatística multivariada}

\subsubsection{Confiabilidade do banco de dados}

O banco de dados utilizado nesta pesquisa apresentou um Alfa de Cronbach da ordem de 0,714 . Assim, garantida a confiabilidade dos dados empíricos, todas as variáveis coletadas em campo foram preservadas para construção das análises.

\subsubsection{Análise Fatorial}

A análise fatorial alcançou quatro fatores por intermédio do método de extração dos componentes principais. Obteve-se o valor de 0,792 para o KMO e 738,466 (000) para o teste de Esfericidade de Bartlett. A tabela 01 apresenta os fatores extraídos e a respectiva carga fatorial de suas variáveis componentes.

Tabela 1: Fatores extraídos

\begin{tabular}{|c|c|c|c|c|c|c|c|c|c|c|c|c|}
\hline $\begin{array}{l}\frac{1}{0} \\
\text { జ் }\end{array}$ & $\begin{array}{l}\frac{0}{0} \\
\frac{\pi}{0} \\
\frac{0}{0} \\
\frac{\pi}{2} \\
\frac{1}{2} \\
\frac{1}{2} \\
\frac{0}{0} \\
\frac{0}{2}\end{array}$ & $\begin{array}{l}\frac{0}{0} \\
\frac{0}{ \pm} \\
\frac{1}{0} \\
0 \\
\frac{1}{0} \\
\frac{0}{2}\end{array}$ & 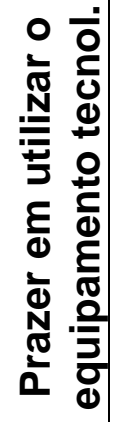 & 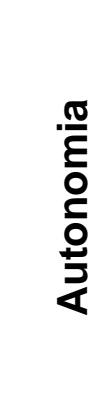 & 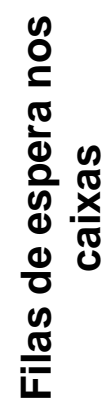 & $\begin{array}{l}\frac{\pi}{0} \\
\frac{c}{d} \\
\frac{0}{0} \\
\frac{0}{\omega} \\
\frac{1}{0} \\
\frac{0}{\pi}\end{array}$ & 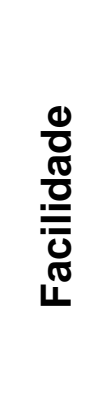 & $\begin{array}{l}\frac{0}{0} \\
\frac{\pi}{0} \\
\frac{0}{8} \\
0 \\
0 \\
0\end{array}$ & $\begin{array}{l}\mathbb{N} \\
\frac{0}{0} \\
\frac{0}{2} \\
\mathbb{N} \\
\end{array}$ & 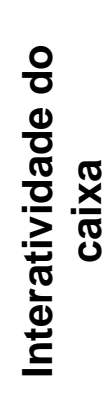 & 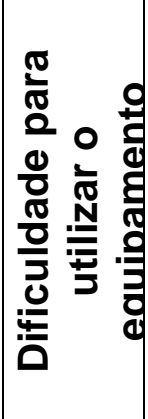 & 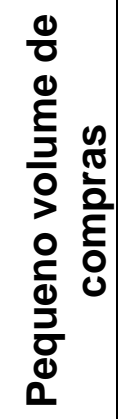 \\
\hline 1 & 0,798 & 0,749 & 0,675 & 0,660 & & & & & & & & \\
\hline 2 & & & & & 0,759 & 0,648 & 0,637 & 0,580 & 0,566 & & & \\
\hline 3 & & & & & & & & & & 0,806 & \begin{tabular}{|l|}
0,757 \\
\end{tabular} & \\
\hline 4 & & & & & & & & & & & & 0,926 \\
\hline
\end{tabular}

Fonte: Autores (2018)

O fator 01 , composto pelas variáveis maior privacidade, maior controle, prazer em utilizar o equipamento tecnológico e autonomia, foi renomeado como "autonomia no atendimento" e apresenta um alfa de Cronbach de 0,752. O fator 02, composto pelas variáveis fila de espera nos caixas tradicionais, maior eficiência, facilidade, comodidade e rapidez, foi renomeado como "eficiência no atendimento" e apresenta um alfa de Cronbach de 0,748. O terceiro fator, composto pelas variáveis interatividade do caixa e nível de dificuldade para utilizar o self-checkout foi renomeado como "facilidade em utilizar o equipamento de selfcheckout" e apresenta um alfa de Cronbach de 0,606. O quarto e último fator é composto exclusivamente pela variável pequeno volume de compras, corroborando com Barreto, Fernandes e Ortuño (2017). Os quatro fatores extraídos, explicam $60,671 \%$ da variância, conforme apontado pela tabela 2. 
Tabela 2: Variância explicada

\begin{tabular}{|c|c|c|c|}
\hline Fator & Autovalor & Variância (\%) & Variância acumulada (\%) \\
\hline 1 & 4,214 & 32,417 & 32,417 \\
2 & 1,580 & 12,154 & 44,571 \\
3 & 1,093 & 8,405 & 52,976 \\
4 & 1,000 & 7,695 & 60,671 \\
\hline
\end{tabular}

Fonte: Autores (2018)

\subsubsection{Clusters}

A análise de clusters foi realizada com o intuito de identificar os diferentes grupos que utilizam o self-checkout. Para tal, foram processados agrupamentos de 2 , 3 e 4 clusters, sendo classificados $199(99,5 \%)$ dos casos. A tabela 3 indica a distribuição dos indivíduos entre os clusters formados.

Tabela 3: Formação dos clusters

\begin{tabular}{|c|c|c|c|c|}
\hline \multirow{2}{*}{ Clusters } & \multicolumn{4}{|c|}{ Quantidade de indivíduos por grupo } \\
\cline { 2 - 5 } & $\mathbf{1}$ & $\mathbf{2}$ & $\mathbf{3}$ & $\mathbf{4}$ \\
\hline $\mathbf{2}$ & 140 & 59 & & \\
\hline $\mathbf{3}$ & 140 & 46 & 13 & 13 \\
\hline $\mathbf{4}$ & 66 & 46 & 74 & 13 \\
\hline
\end{tabular}

Fonte: Autores (2018)

Evidencia-se a presença de um conjunto composto por 13 indivíduos que tanto nas formações com 3 ou 4 cluster continua independente frente as possibilidades de agrupamento. Desta forma, visando uma caracterização mais heterogênea entre os grupos, foi adotada a composição com 4 clusters. Portanto, $\mathrm{O}$ grupo 01 é composto por $66(33,2 \%)$ indivíduos, o grupo 02 por $46(23,1 \%)$, o grupo 03 por $74(37,2 \%)$ e o grupo 04 por $13(6,5 \%)$ indivíduos.

\subsubsection{Análise Discriminante}

Após a definição dos clusters, realizou-se análise discriminante para identificar as variáveis que diferenciam os grupos. Os resultados demonstram que nove variáveis discriminam os quatro clusters adotados: fila de espera nos caixas tradicionais, autonomia, interatividade do caixa, maior eficiência, maior controle, nível de dificuldade para utilizar o self-checkout, rapidez, pequeno volume de compras e facilidade de utilização do equipamento. Foram geradas três funções discriminantes, alcançando os respectivos valores de correlação canônica: 0,887, 0,811 e 0,580 . Assim, pode-se afirmar que as funções discriminam, respectivamente, $78,677 \%, 65,772 \%$ e $33,640 \%$ da variância. Os resultados da análise discriminante revelam ainda que, $87,4 \%$ dos casos foram classificados corretamente. 


\subsubsection{ANOVA}

Devido a necessidade de compreender as diferenças entre os quatro clusters adotados, utilizou-se a análise de variância (ANOVA) para comparação das médias relacionadas às variáveis apontadas pela análise discriminante, os valores observados podem ser verificados na tabela 4.

Tabela 4: ANOVA

\begin{tabular}{|c|c|c|c|c|}
\hline \multirow{2}{*}{ Variável } & \multicolumn{4}{|c|}{ Média verificada } \\
\cline { 2 - 5 } Filas de espera dos caixas tradicionais & 4,833 & 4,934 & 5,000 & 3,076 \\
\hline Autonomia & 4,742 & 2,782 & 4,635 & 4,153 \\
\hline Interatividade do equipamento & 2,909 & 2,847 & 3,824 & 2,846 \\
\hline Eficiência do equipamento & 4,439 & 3,173 & 4,486 & 2,615 \\
\hline Facilidade de utilização do equipamento & 4,424 & 3,282 & 4,959 & 2,923 \\
\hline Rapidez & 4,772 & 4,260 & 4,973 & 3,769 \\
\hline
\end{tabular}

Fonte: Autores (2018)

A partir dos valores atribuídos por cada grupo às variáveis destacadas acima propõem-se a seguinte nomenclatura para os clusters apresentados:

- Grupo 1: Os independentes, valorizam a autonomia, o controle e a eficiência.

- Grupo 2: Os apressados, valorizam rapidez do self-checkout.

- Grupo 3: Os entusiasmados, consideram que o self-checkout é muito melhor que os caixas tradicionais em diversos aspectos

- Grupo 4: Os “indiferentes”, não veem grandes diferencias na utilização do self-checkout.

A identificação do Grupo 2, "Os apressados", corrobora com o trabalho de Barreto, Fernandes e Ortuño (2017), que evidencia a escassez de tempo da sociedade contemporânea, grupo de indivíduos que, consequentemente, busca por recursos capazes de tornarem seus afazeres mais eficientes, a fim de lograr suas múltiplas funções diárias como estudar, trabalhar, além das demais atividades sociais. Com exceção do Grupo 4, "Os indiferentes", percebe-se que todos os demais representam consumidores que trazem consigo motivações em utilizar o equipamento de self-checkout, resultado que se mostra consonante com os achados de Fernandes e Pedroso (2017) por expor que na ação em utilizar o equipamento de autoatendimento, o consumidor deve se sentir motivado e reconhecer benefícios em tal experiência.

Nota-se que independente da experiência prévia na utilização do selfcheckout não existiram grandes problemas quanto a interatividade de operação do equipamento. Apenas 10 clientes $(5,0 \%$ do total de respondentes) afirmaram que não tiveram a expectativa atendida, contudo, $70 \%$ destes continuam recomendando a utilização do terminal de autoatendimento; além disso, $80 \%$ recomendam 0 RELNA, Belo Horizonte - MG, Brasil, v.24, n.I, p.41-57, Jan. - Mar. 2019 - ISSN 2179-8834 
estabelecimento varejista à outras pessoas. Quanto ao atendimento das necessidades, 5 clientes $(2,5 \%$ do total de respondentes) declararam que uma experiencia não satisfatória, contudo, $60 \%$ destes continuam recomendando a utilização do terminal de autoatendimento; e 80\% recomendam o estabelecimento varejista à outras pessoas. Os resultados apresentados corroboram com Fernandes e Pedroso (2017), demonstrando que a qualidade e satisfação percebida na utilização dos terminais de autoatendimento têm efeitos diretos na intenção de retorno dos clientes e fidelidade nos estabelecimentos comerciais. Não foi observada nenhuma relação entre a experiência percebida pelo cliente e a avaliação do equipamento de autoatendimento; desta forma, os resultados se opõem ao trabalho de Djelassi, Diallo e Zielke (2018) e minimizam os potencias desvios resultantes de experiencias negativas quanto ao autoatendimento. A tabela 5 caracteriza os 4 grupos frente as observações realizadas e as distribuições predominantes.

Portanto, nota-se que, na maioria absoluta dos casos o equipamento atendeu às necessidades e expectativas previstas, demonstrando a implicação da utilização do terminal de self-checkout e a satisfação dos consumidores.

Tabela 5: Resumo dos clusters adotados

\begin{tabular}{|c|c|c|c|c|c|}
\hline & & & & & \\
\hline Gênero & \multirow{5}{*}{ 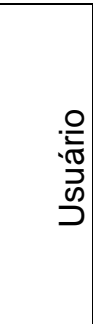 } & Feminina & Masculino & Feminino & Masculino \\
\hline Faixa etária & & $\begin{array}{l}18 \text { a } 45 \\
\text { anos }\end{array}$ & 18 a 45 anos & 18 a 45 anos & 18 a 45 anos \\
\hline Número de filhos & & Nenhum & Nenhum & Nenhum & Nenhum \\
\hline Estado Civil & & Solteiro & Solteiro & Solteiro & Solteiro \\
\hline Escolaridade & & $\begin{array}{l}\text { Superior } \\
\text { completo }\end{array}$ & $\begin{array}{l}\text { Pós- } \\
\text { graduação } \\
\text { completa }\end{array}$ & $\begin{array}{l}\text { Superior } \\
\text { incompleto }\end{array}$ & $\begin{array}{l}\text { Superior } \\
\text { Completo }\end{array}$ \\
\hline $\begin{array}{r}\text { Frequência de uso de } \\
\text { computador, celular e } \\
\text { internet }\end{array}$ & \multirow{2}{*}{ 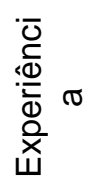 } & Diariamente & Diariamente & Diariamente & Diariamente \\
\hline $\begin{array}{r}\text { Experiência anterior no uso } \\
\text { do self-checkout }\end{array}$ & & Sim & Sim & Não & Não \\
\hline $\begin{array}{r}\text { Qualidade da orientação } \\
\text { recebida para uso do self- } \\
\text { checkout }\end{array}$ & \multirow{6}{*}{ 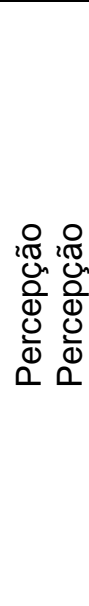 } & Suficiente & $\begin{array}{c}\text { Sem } \\
\text { orientação }\end{array}$ & Suficiente & $\begin{array}{c}\text { Sem } \\
\text { orientação }\end{array}$ \\
\hline $\begin{array}{r}\text { Comparação com os caixas } \\
\text { tradicionais }\end{array}$ & & É melhor & É melhor & É melhor & Indiferente \\
\hline $\begin{array}{r}\text { O equipamento de self- } \\
\text { checkout atendeu as } \\
\text { expectativas e as } \\
\text { necessidades }\end{array}$ & & Sim & Sim & Sim & Sim \\
\hline Recomenda o self-checkout & & Sim & Sim & Sim & Sim \\
\hline $\begin{array}{l}\text { O supermercado demonstra } \\
\text { ser uma empresa inovadora }\end{array}$ & & $\begin{array}{c}\text { Concordo } \\
\text { parcialmente }\end{array}$ & Indiferente & $\begin{array}{l}\text { Concordo } \\
\text { totalmente }\end{array}$ & Indiferente \\
\hline $\begin{array}{r}\text { Recomenda outras pessoas } \\
\text { a comprarem no } \\
\text { supermercado }\end{array}$ & & Sim & Sim & Sim & Sim \\
\hline
\end{tabular}


Gustavo Nunes Maciel, Jasé Augusta Dliveira, Gustava Clemente Valadares, Paulo Henrique Montagnana Vicente Leme,

Daniel Carvalho Rezende

Tabela 5: Resumo dos clusters adotados (continuação)

\begin{tabular}{|c|c|c|c|c|c|}
\hline & & Grupo 1 & \multirow{2}{*}{$\begin{array}{c}\text { Grupo } 2 \\
\text { Muito } \\
\text { importante }\end{array}$} & \multirow{2}{*}{$\begin{array}{c}\text { Grupo 3 } \\
\text { Muito } \\
\text { importante }\end{array}$} & \multirow{2}{*}{$\begin{array}{c}\text { Grupo } 4 \\
\text { Indiferente }\end{array}$} \\
\hline $\begin{array}{c}\text { Filas de espera nos caixas } \\
\text { tradicionais }\end{array}$ & \multirow{7}{*}{ 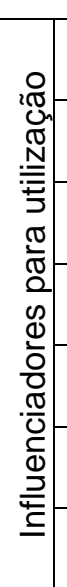 } & $\begin{array}{c}\text { Muito } \\
\text { importante }\end{array}$ & & & \\
\hline Autonomia & & $\begin{array}{l}\text { Muito } \\
\text { importante }\end{array}$ & Indiferente & $\begin{array}{c}\text { Muito } \\
\text { importante }\end{array}$ & Importante \\
\hline $\begin{array}{l}\text { Interatividade do } \\
\text { equipamento }\end{array}$ & & $\begin{array}{l}\text { Bastante } \\
\text { intuitivo }\end{array}$ & $\begin{array}{c}\text { Bastante } \\
\text { intuitivo }\end{array}$ & $\begin{array}{l}\text { Totalmente } \\
\text { intuitivo }\end{array}$ & $\begin{array}{l}\text { Bastante } \\
\text { intuitivo }\end{array}$ \\
\hline Maior eficiência & & Importante & Indiferente & $\begin{array}{c}\text { Muito } \\
\text { importante }\end{array}$ & Indiferente \\
\hline Maior controle & & Importante & $\begin{array}{l}\text { Pouco } \\
\text { importante }\end{array}$ & $\begin{array}{c}\text { Muito } \\
\text { importante }\end{array}$ & Indiferente \\
\hline Rapidez & & $\begin{array}{c}\text { Muito } \\
\text { importante }\end{array}$ & Importante & $\begin{array}{c}\text { Muito } \\
\text { importante }\end{array}$ & Importante \\
\hline Facilidade de utilização & & Importante & Indiferente & $\begin{array}{c}\text { Muito } \\
\text { importante }\end{array}$ & Indiferente \\
\hline
\end{tabular}

Fonte: Autores (2018)

\section{Considerações finais}

A acirrada concorrência percebida no mercado contemporâneo aliada ao processo desenfreado de inovações tecnológicas trazem às organizações atuantes um grande desafio de sobrevivência e competitividade (TRIERWEILLER et al., 2017). De forma a combater com as dificuldades encontradas, muitos gestores buscam alternativas tecnológicas que visam reduzir os custos, adquirir valor e melhorar a satisfação do cliente (DEMIRCI OREL; KARA, 2014). Entre elas estão os serviços de autoatendimento - Self-Checkout.

Com a adoção deste recurso tecnológico, os gestores têm em suas mãos a oportunidade de atender diferentes públicos de forma diferenciada e inovadora (FERNANDES; PEDROSO, 2017). Consequentemente, as organizações poderão buscar a satisfação do consumidor que vem sendo compreendida como um fator de sucesso, alinhando seus processos às necessidades percebidas de seu público (OLIVER, 1997).

Neste sentido, oferecer o serviço inovador e de qualidade se torna um valioso instrumento que contribui para a satisfação dos clientes, estes que cada vez mais buscam serviços diferenciados e com alto nível de exigência (ZEITHAML; BITNER, 2003). Nota-se que os consumidores não estão em busca de apenas um produto ou serviço, mas de uma experiência de compra que seja igual ou além de suas expectativas e anseios (TRIERWEILLER et al., 2017). Nesta linha, a utilização do autoatendimento self-checkout pode ser muito bem empregada em uma sociedade que cada vez mais compartilha seus momentos e ações com a presença de algum artefato tecnológico específico.

No esforço de atingir o objetivo geral da pesquisa, investigar o perfil e os fatores relacionados à satisfação dos consumidores que optam por utilizar o serviço de autoatendimento empregado por uma rede supermercadista da cidade de Lavras, no sul de Minas Gerais, pode-se considerar que os resultados trouxeram algumas respostas para as inquietações dos pesquisadores. A partir do alto índice relacionado ao alcance das expectativas e necessidades do consumidor, REULA, Bela Horizonte - MG, Brasil, v.24, n.l, p.41-57, لan. - Mar. 2019 - ISSN 2179-8834 
constatados pela pesquisa, torna-se viável afirmar que a experiência com o autoatendimento gerou satisfação para grande parte dos clientes, que ainda demonstraram, em grande parte, preferi-lo em relação ao caixa convencional.

Quanto aos objetivos específicos, deve-se considerar que: (I) a investigação em torno da experiência do consumidor com o self-checkout foi realizada abordando diversos aspectos como a interatividade, a intimidade com recursos tecnológicos, a faixa etária, grau de formação, entre outros, com o intuito de explorar ao máximo as características do público e suas percepções; (II) a verificação dos fatores motivacionais do consumidor ao utilizar o recurso tecnológico de autoatendimento foi realizada constatando que, entre as diversas causas apresentadas, os fatores maior comodidade, maior rapidez e a fila percebida nos caixas tradicionais assumiram a posição de maior relevância aos entrevistados; e (III) a confrontação com a literatura permitiu discutir considerada parte dos resultados apresentando uma maior quantidade de pontos em comum do que aqueles que destoaram.

As contribuições da presente pesquisa são percebidas em dois distintos ambientes. Primeiramente, no âmbito gerencial, tem-se o potencial de contribuir com os gestores na implementação de equipamentos de autoatendimento, de modo que a sua utilização esteja alinhada aos anseios dos consumidores. Em segundo, no âmbito acadêmico da ciência administrativa, este estudo pretende contribuir para mitigar uma lacuna teórica sobre tema investigado ao discutir as implicações do serviço de autoatendimento na satisfação e lealdade dos consumidores.

Algumas limitações foram percebidas na pesquisa; entre elas está o emprego da abordagem transversal utilizada, que investiga em um só momento o objeto. Além disso, percebe-se que a análise de uma organização somente limita os achados e, ainda, pode produzir certo viés.

Pesquisas futuras para o tema, como a oportunidade de realizar a pesquisa em contextos diferentes que também optaram pela utilização do autoatendimento no mesmo segmento, trará novos achados e potenciais comparações, até mesmo propondo um estudo multicasos. Por fim, sugere-se o emprego de estudos longitudinais, que possam avaliar a percepção dos consumidores e as ações gerenciais para a sua manutenção.

\section{Referências}

$\begin{array}{llcr}\text { ALLIED MARKET RESEARCH. World self services technologies market e } & \text { tech } \\ \text { Opportunities } & \text { and forecasts. } 2015 . & \text { Disponível } & \text { em: } \\ \text { <https://www.alliedmarketresearch.com/self-services-technologies-market> } & \text { Acesso }\end{array}$ em 25 mar. 2019.

ANDERSON, E. W., FORNELL, C. LEHMANN, D. R. Customer satisfaction, market share, and profitability: Findings from Sweede. Journal of Marketing, v. 58, n. 3, p. 53-66, 1994.

BALBIM JUNIOR, A.; BORNIA, A. C. Proposta de um instrumento de medida para avaliar a satisfação de clientes de bancos utilizando a Teoria da Resposta ao Item. Gestão \& Produção, v. 18, n. 3, p. 541-554, 2011. 
BARRETO FERNANDES, F. A.; ORTUÑO, B. H. Usability and User-Centered Design - User Evaluation Experience in Self-Checkout Technologies. Proceedings Systems \& Design 2017. Anais.Valencia: Universitat Politècnica València, 30 nov. 2017Disponível em: <http://ocs.editorial.upv.es/index.php/SD/SD2017/paper/view/6634>

COLLIER, J. E.; BREAZEALE, M.; WHITE, A. Giving back the "self" in self service: customer preferences in self-service failure recovery. Journal of Services Marketing, v. 31, n. 6, p. 604-617, 2017.

CONSIDINE, E.; CORMICAN, K. Self-service Technology Adoption: An Analysis of Customer to Technology Interactions. Procedia Computer Science, v. 100, p. 103109, 2016.

DEMIRCI OREL, F.; KARA, A. Supermarket self-checkout service quality, customer satisfaction, and loyalty: Empirical evidence from an emerging market. Journal of Retailing and Consumer Services, v. 21, n. 2, p. 118-129, 2014.

DJELASSI, S.; DIALLO, M. F.; ZIELKE, S. How self-service technology experience evaluation affects waiting time and customer satisfaction? A moderated mediation model. Decision Support Systems, 2018.

FERNANDES, T.; PEDROSO, R. The effect of self-checkout quality on customer satisfaction and repatronage in a retail context. Service Business, v. 11, n. 1, p. 6992, 2017.

FORNELL, C., JOHNSON, M. D., ANDERSON, E. W., CHA, J., BRYANT, B. E.. The American customer satisfaction index: nature, purpose, and findings. The Journal of Marketing, p. 7-18. 1996

FREUND, J. E.; SIMON, G. A. Estatística aplicada. 9. ed. Porto Alegre: Bookman, 2000

GIL, A. C. Métodos e técnicas de pesquisa social. 6. ed. São Paulo: Atlas, 2008.

GREWAL, D; LEVY, M. Retailing research: past, present, and future. Journal of Retailing, v. 83, n. 4, p. 447- 464, 2007.

GRONROOS, C. Marketing: gerenciamento e serviços - a competição por serviços na hora da verdade. Rio de Janeiro: Campus, 1993.

HAIR, J. J. F. et al. Análise multivariada de dados (6a. ed.). [s.I.] Grupo A Bookman, 2009.

INMAN, J. J.; NIKOLOVA, H. Shopper-Facing Retail Technology: A Retailer Adoption Decision Framework Incorporating Shopper Attitudes and Privacy Concerns. Journal of Retailing, v. 93, n. 1, p. 7-28, 2017. 
JOHNSON, V. L. et al. The Impact of Consumer Confusion on Mobile Self- Checkout Adoption The Impact of Consumer Confusion on Mobile Self-Checkout Adoption. Journal of Computer Information Systems, v. 00, n. 00, p. 1-11, 2019.

KAZANCOGLU, I.; KURSUNLUOGLU YARIMOGLU, E. How food retailing changed in Turkey: spread of self-service technologies. British Food Journal, v. 120, n. 2, p. 290-308, 2018.

KOKKINOU, A.; CRANAGE, D. A. International Journal of Hospitality Management Using self-service technology to reduce customer waiting times . International Journal of Hospitality Management, v. 33, p. 435-445, 2013.

LEE, H.-J.; LYU, J. Exploring factors which motivate older consumers' self-service technologies (SSTs) adoption. The International Review of Retail, Distribution and Consumer Research, v. 29, n. 2, p. 218-239, 15 mar. 2019.

LEE, H.; LYU, J. Computers in Human Behavior Personal values as determinants of intentions to use self-service technology in retailing. Computers in Human Behavior, v. 60, p. 322-332, 2016.

LENG, H. K.; WEE, K. N. L. An examination of users and non-users of self-checkout counters. International Review of Retail, Distribution and Consumer Research, v. 27, n. 1, p. 94-108, 2017.

MALHOTRA, N. K. Pesquisa de marketing: uma orientação aplicada. Bookman Editora. 2012

MEUTER M. L.; OSTROM A.L.; ROUNDTREE R.; BITNER M.J.; Self-service technologies: understanding customer satisfaction with technology-based service encounters, Journal of Marketing v.1, n.64, p. 50-64, 2000.

MUKERJEE, Hory Sankar; DESHMUKH, G. K.; PRASAD, U. Devi. Technology Readiness and Likelihood to Use Self-Checkout Services Using Smartphone in Retail Grocery Stores: Empirical Evidences from Hyderabad, India. Business Perspectives and Research, v. 7, n. 1, p. 1-15, 2019.

OLIVEIRA, A. S. DE et al. Influência do Crowding na lealdade mediado pela satisfação do consumidor em processos de compras no varejo. Revista de Administração da UFSM, v. 10, n. 4, p. 614-631, 2017.

OLIVER, R. L. Satisfaction: a behavioral perspective on the consumer. New York: McGraw Hill, 1997.

PRADO, P. H. M; MARCHETTI, R. Excelência em supermercados: a dimensão da satisfação do consumidor. Revista de Administração da Universidade de São Paulo, v. 32, n. 2, 1997. 
TRIERWEILLER, A. C. et al. Satisfação de clientes utilizando a perspectiva descritiva e o modelo logístico. Iberoamerican Journal of Industrial Engineering, v. 9, n. 17, p. 190-206, 2017. 